monoclonal eliminates the possibility that their nonspecificity is apparent rather than real, and that the cells are really producing a very large number of different specific factors.

A similar collection of hybridomas producing helper factors would be a great asset. The biochemical elucidation of helper factors lags well behind that of suppressors. There is now evidence for idiotype-bearing, antigen-specific factors which replace $T$ cell help in the production of antibodies against phosphorylcholine (M. Feldman, University College London) and $(T, G)-A--L$ (ref .6) but the relationship of such specific factors to nonspecific helper factors remains unclear. J.D. Watson (University of California at Irvine), for example, reported that $T$ cell growth factor(s) from supernatant of spleen cells treated with concanavalin A would act both as a nonspecific growth factor and as a nonspecific helper factor in antibody production or the generation of cytotoxic $T$ cells; but if microcultures of ten or so cells are expanded with the use of the growth factor, their supernatants are active only on some cells. This experiment, which is similar to that of Waldmann and Lefkowitz ${ }^{7}$ suggests that 'nonspecific' growth factors from concanavalin A supernatants may be mixtures of antigen or idiotype-specific factors; it remains to be seen whether such mixtures include a genuinely nonspecific $T$ cell growth factor.

MHC restriction, which limits $T$ cell recognition of viral antigens to infected cells bearing the same $\mathrm{MHC}$ antigens, can be seen as a special case of antigen specificity applied to $\mathrm{T}$ cells. It has now become clear that, like any other kind of antigen recognition, $\mathrm{MHC}$ restricted recognition is subject to cross-reaction. Cross-reactions have been demonstrated in experiments in which allogeneic virusinfected cells are killed by cytotoxic cells depleted of alloreactivity by passage through the appropriate mouse strain (J.R. Bennink, University of Philadelphia). The cross-reaction may not be reciprocal: for example, b haplotype cells may kill infected $\mathrm{k}$ haplotype targets while $\mathrm{k}$ cells will not kill infected $\mathrm{b}$ targets ${ }^{8}$.

The converse can also occur: certain combinations of haplotype and virus can fail to elicit a cytotoxic response even when MHC restriction requirements are met. Much of the interest in such failures of recognition is of course due to their possible bearing on susceptibility to disease in man. A McMichael (Oxford University) reported that human lymphocytes of certain haplotypes will not show MHCrestricted killing in association with influenza virus antigens, whereas others do. Similarly, S. Shaw (US National Institute of Health) reported that some HLA haplotypes elicit much stronger antiinfluenza cytotoxic responses than others.

Miranda Robertson is an Associate Editor of Nature.
These data lend support to the proposal that the immense polymorphism of the MHC antigens is due to the massive selective advantage of heterozygosity: if the combination of one parent's MHC antigen and virus does not elicit a response, the other parent's may (indeed this has been shown ${ }^{9}$ ).

However, this begs the question of why antigens that fail to elicit an immune response should survive in the population. The answer may lie in the mechanism of selective recognition. It is now clear that some combinations of MHC antigen and virus cross-react with alloantigens ${ }^{10}$ : it is thus highly likely that the combination of some viral antigens with self antigens will crossreact with other self antigens. Since any cell reacting strongly against self must be eliminated, it follows that cells reacting against certain combinations of self and virus will sometimes be eliminated too.

While it is clear that to avoid autoimmune reactions $T$ cells with highaffinity receptors for self must be eliminated, it has been suggested that receptors with weak affinity for self antigens are the basis for MHC restricted recognition ${ }^{11}$. Tentative evidence in support of this theory was presented by $\mathbf{H}$. Wigzell (Uppsala University), who has used an anti-idiotypic serum raised against immune $T$ cells specific for an allogeneic antigen to extract the $T$ cell receptor for that antigen. He then labelled internally the allogeneic antigen, the self antigen of the immune rat and a third-party antigen, and ran them on Sepharose columns to which the receptor was bound. Three labelled polypeptides from the third-party control passed straight through the column; two chains from the cells of the immunising strain were stopped; and one chain from the self strain was retarded.

Wigzell interprets this as evidence not only for weak binding of alloreactive receptors to self, but also as evidence that different polypeptides are involved in the recognition of self MHC and allo- or viral determinants. It is still a matter of disupte whether $T$ cell receptors 'see' virus and MHC antigen as a complex, with only one receptor for both ('altered self'), or whether they see the two antigens with different receptors ('dual recognition') ${ }^{12}$. Attempts to resolve this question at a cellular level are all open to doubt: it is probable that only biochemistry can produce the answer, and Wigzell's experiment may be a first step.

\footnotetext{
I Eichmann, K. Eur. J. Immun. 5, $511-517$ (1975),

2 Eichmann, X. \& Rajewski, K. Eur. J. Immun. 5, 661-666 (1975).

Q1975).

${ }^{4}$ Ben-Nevia et al. Eur. J. Immun. 8, 797 (1978)

Taniguchi, M. et al. Nature (in the press).

6 Mozes, E. \& Haimovich, J. Nature 278, $56-57$ (1979).

Lefkowitz, 1.\& Waldmann, H. Immunology 32, 915 (1977).

8 .

9

10 haw, S. \& B.ddison, W.E. J. exp. Med. 149, S65-STS (1979)

We.

Proc. natn, Acad. Sci. U.S.A. 75, 5145-5149 (1978).

I1 Janeway, C.A. Wigzell, H. \& Binz, H. Scand. J. Immun. 5 993-1001 (1976).
}

\section{Ecology of photosynthesis}

\section{from Peter $D$. Moore}

Resource partitioning in plants is less well documented than among animals and this is not surprising, for the resources on which plants depend are fewer and adaptations which could be referred to as partitioning are generally more subtle than is the case with animals. This is probably why plant ecophysiologists have eagerly grasped the opportunities presented by the gradual exposition of the extent of the $\mathrm{C}_{4}$ and crassulacean acid metabolism (CAM) photosynthetic adaptations among plants. It is well established that both of these modifications to the basic $\mathrm{C}_{3}$ Calvin photosynthetic system are associated with certain ecologically selective advantages in conditions of high temperature, high light intensity and water stress. This has led to many investigations into the biogeographical significance of the adaptations, mainly by mapping their distributions on a continental scale (see Nature 272, 400; 1978). Information is now accumulating concerning the ecological value of such adaptations in the local composition of plant communities.

To search for correlations between the success of one particular type of photosynthetic system and an environmental gradient, such as aridity or elevation, is not new. In 1974, Mooney, Troughton and Berry (Carnegie Inst. Ybk. 73,793 ; 1974) reported a survey they had conducted along the coastal strips of California and Chile, where the climate shifts from a Mediterranean to a desert type with decreasing latitude. They found in both locations that increasing aridity was associated with an increasing proportion of CAM plants in the flora. Among $\mathrm{C}_{3}$ plants, there was a change from evergreen to drought-deciduous forms, but $\mathrm{C}_{4}$ plants were virtually absent from all stations. They explain this as a consequence of the low temperatures prevailing at the times of water availability.

More recent work in the cold winter deserts of Utah by Caldwell, White, Moore and Camp (Oecologia, Berl, 29, 275; 1977) has also shown that such $\mathrm{C}_{4}$ species as Atriplex confertifolia fail to achieve many of the advantages normally associated with the $\mathrm{C}_{4}$ system when they commence growth in the cool of spring. They may, however, still match the growth of their $\mathrm{C}_{3}$ competitors and gain the advantage of a longer growing season as summer aridity sets in. It is difficult, therefore, to explain the lack of $\mathrm{C}_{4}$ species in the Californian and Chilean coastal deserts.

Eickmeier has now published the results of a similar environmental gradient study from Texas (Photosynthetica 12, 290;

Peter D. Moore is a Senior Lecturer in the Department of Plant Sciences, King's College, London. 
1978). Of 88 non-herbaceous species found along a 1,300-m elevation gradient, 22 were CAM, three were $\mathrm{C}_{4}$ and the remaining 63 were $C_{3}$. Ten samples were taken at $150-\mathrm{m}$ altitude intervals; density, frequency and area cover values were used to determine an elevation. The main peak in the importance of CAM species was found to occur at low altitude, that of $\mathrm{C}_{3}$ species at higher altitudes and the $\mathrm{C}_{4}$ peak was intermediate between the other two. Eickmeier considers this sequence to reflect both the adaptational significance of the physiological variations and also the respective responses to biotic, competitive factors. For example, the CAM plants are most suited to the low altitude, arid environment, where competitive interplay with other plant species may be regarded as minimal (but see Nature op. cit). $\mathrm{C}_{3}$ plants, he claims are evolutionarily attuned to competitive situations with low abiotic stress; $C$ plants can cope with both, having potentially high productivity and the importance value for each species at each

capacity to withstand water stress.

The greater competitive success of $C_{3}$ species in higher altitude, more mesic conditions in the tropics has now been demonstrated by Tieszen, Senyimba, Imbamba and Troughton (Oecologia, Berl. 37, 337; 1979) along an altitudinal and moisture gradient in Kenya. They have found that nearly all the grass species of the low altitude grasslands in Kenya are of a $\mathrm{C}_{3}$ photosynthetic type. Some grass tribes, such as Paniceae and Andropogoneae (both exclusively $\mathrm{C}_{4}$ ) were most frequently encountered at intermediate altitudes. The exclusively $\mathrm{C}_{3}$ tribes (such as Festuceae, Aveneae and Agrostideae) were found only at high altitude. No $\mathrm{C}_{3}$ species were found below an altitude of $2,000 \mathrm{~m}$ and no $\mathrm{C}_{4}$ species above $3,000 \mathrm{~m}$.

Evidently the complex adaptational significance of $\mathrm{C}_{3}, \mathrm{C}_{4}$ and CAM photosynthetic systems is profoundly influential on the floristic composition of plant communities both on a biogeographic and an ecological scale.

\section{Viral pathogenesis and virulence}

\section{from D.A.J. Tyrrell}

IT is easy to say "The influenza virus causes influenza", while having really no scientific explanation of many of the signs and symptoms of the disease, for instance the headache or the depressed white blood cell count. This meeting* would have jolted anyone out of such a complacent view and also gave some good examples of how patient, thoughtful research is building a more complete picture of what happens in some virus infections - how the virus enters and spreads through the body, enters cells, disturbs their function, interacts with the host defence mechanisms (such as interferon or humoral or cellular immunity) and disturbs normal body functions to produce what we recognise as a clinical disease.

What seem in the laboratory to be very similar viruses may nevertheless produce either different diseases or similar diseases by different methods. There was for instance a session on coronaviruses, which all look the same and are similar in biochemical composition. Yet the different coronaviruses are specific to particular species - mouse coronaviruses never seem to affect man or vice versa, for example and sometimes even to a very limited variety of cells in one species. P.A. Bachmann (World Health Organization, Munich) described how the coronavirus of transmissible gastroenteritis of pigs (TGE) affects only the differentiated enterocytes of the villi of the small intestine which may almost disappear. The crypt cells, * A symposium on Mechanisms of Viral Pathogenesis and organised by P.A. Bachmann. however, survive and migrate up to cover the villi again, but while they are doing this and differentiating into mature enterocytes, there are profound physiological disturbances - lack of absorption, excess secretion, lack of digestive function (for instance the splitting of lactose by disaccharidase) leading to marked diarrhoea, which can kill a piglet from dehydration unless it is treated. On the other hand, another pig coronavirus causes vomiting and wasting disease. M. Pensaert and K.L. Andries (State University of Ghent) described how this virus affects the upper respiratory tract and lung, the tonsil and the jejunum; it affects the cells of the plexus of Auerbach and Meissner in the gut and seems to spread from them. It spreads through the nervous system to the brain stem and later to the spinal cord and the rest of the brain. All this was established in great detail by painstaking serial virus assay and immunofluorescent microscopy of experimentally infected animals. This suggests how the disease develops - probably the virus spreads from the respiratory tract and gut along peripheral nerves to the brain stem where the lesions give rise to vomiting, while the damage to neurones in the plexuses prevents normal peristalsis, leading to wasting. Furthermore, knowing that the virus invades the nervous system widely, it is not surprising that a virtually identical virus has been recovered from pigs diagnosed as having encephalitis, and so was named haemagglutinating encephalitis virus.

Explanations of these important and mysterious tropisms of viruses must lie at the molecular level, in the functioning of the peptides and nucleic acids of which viruses are made. B. Fields (Harvard Medical School) gave an example of what can be done, when he described his study of two pairs of genes, represented by the RNAs $\mathrm{S} 1$ and M 2 of reoviruses types 1 and 3 . He studied parent viruses and a collection of recombinants (or reassortants) and showed that S1 nucleic acid specifies the haemagglutinin protein of the virus and that this is responsible for the ability to agglutinate red cells and for serological specifity. It is also responsible for cell tropism in the animal, so that the type 3 virus causes a necrotising encephalitis due to a specific attack on the neurones. It also determines the extent to which the virus inhibits DNA synthesis when absorbed to cells. M2 RNA on the other hand, which is abundant on the surface of the virus particle determines whether the virus resists inactivation by chymotrypsin. M2 RNA from type 3 makes the virus resistant to host enzymes and hence able to infect when given by mouth. Discussion of this work showed that the next step was to determine at which site on the surface of the neurone the virus attached. This is probably a part of the chemical matrix which has some other physiological function characteristic of neurones. Reassortants were also made which were infectious when given by mouth, like type 1 , and caused central nervous system disease, like type 3 , and so, by the oral route, were more pathogenic than either parent. This type of manipulation confirms earlier conclusions about the functions of the genes.

It is very difficult to evaluate the way in which the host defences interact with the virus, sometimes preventing its multiplication, sometimes causing immunological damage to infected tissue. For instance, P.A. Neighbour and B.R. Bloom (Albert Einstein College of Medicine, New York) described some of their work which started when they analysed why the lymphocytes of patients with multiple sclerosis show an unusual reactivity to measles virus; they tracked down the fault to a defect in the ability of their lymphocytes to produce type 1 interferon (Neighbour \& Bloom Proc. natn. Acad. Sci. U.S.A. 76, 476; 1979).

The success of the meeting was obvious, and there were many other interesting papers besides those mentioned here, including whole sessions on herpes viruses and rhabdoviruses. There were clear signs that pathogenesis not only holds scientific interest, but may be a source of practically useful knowledge: vaccines and antiviral treatments do not always work - sometimes because we guess too much and know too little about the process by which viruses produce disease.

D.A.J. Tyrrell is in the Division of Communicable Diseases, Clinical Research Centre, Harrow. 